\title{
Evaluation of the Effect of Corrected Nutrition on Reduction of Stress in Gestational Diabetes
}

\author{
Mohtaram Andalib ${ }^{1}$, Ramazan Hassanzadeh ${ }^{*}$, Farhad Mashayekh Bakhshi ${ }^{2}$
}

\begin{abstract}
Objectives: Gestational diabetes is defined as impaired glucose tolerance during early pregnancy. Many evidence showed that proper regimen and physical activity have inhibitory effect on incidence of gestational diabetes. In many guidelines, it is advised for performing screening tests for gestational diabetes in early stages of pregnancy and post-partum consulting for changes in life style. Materials and Methods: In this study, 40 pregnant women with gestational diabetes were enrolled. In this study, 40 pregnant women with gestational diabetes were enrolled. In this research an experimental design with pre-test and post-test and control group, in the form of a written program to reduce stress among pregnant women was carried out. The effectiveness of the educational principles of nutrition in diabetes program to reduce the stress was evaluated in experimental group and control group that did not receive any training.

Results: The results indicate the effect of reducing stress in trained pregnant women. Also the effect of nutrition training on blood sugar, the results indicate a reduction in $\mathrm{HbA1C}$ levels compared to the control group.

Conclusion: Due to the impact of education on reducing maternal stress and glycemia control and pregnancy, it is recommended to target the education of proper nutrition and self-care in pregnant women even in patient with diabetes type 2 , as a simple and cheap therapeutic procedure.

Keywords: Blood glucose, Diabetes mellitus, Education, Gestational, Nutritional status, Self-care, Stress, Women
\end{abstract}

\section{Introduction}

Gestational diabetes refers to the carbohydrate intolerance that is initially diagnosed during pregnancy and is observed in about $7 \%$ to $14 \%$ of pregnancies (1). Gestational diabetes ends immediately after delivery; however, $20 \%$ of women with this condition will develop type 2 diabetes during the 9 years following labor (2). Physiological and psychological changes that occur during pregnancy can strengthen mother's emotional stability or cause psychopathological disorders such as stress and anxiety (3). Stress during pregnancy, labor and lactation can be associated with adverse outcomes such as gestational diabetes, abortion, nausea, vomiting, pre-eclampsia (high blood pressure during pregnancy), preterm labor, low birth weight, immune system suppression, and consequently, increased risk of infections and some degrees of mental disorders following labor (4). The stress phenomenon generally refers to psychological responses to mental and physical needs and threats (stressors) (5).

According to Cohen's definition, when environmental needs, whether internal or external or real or imaginary, pressurizes the individual or exceed the individual's tolerance, it causes psychological and biological changes as a result of the stress and exposes the individual to the risk of various diseases (6). According to the World Health Orga- nization (WHO), stress is the second leading threat to human health worldwide (7). It has been shown in the past two decades that stress causes unfavorable consequences in people's health through psychological and biological changes (6). Gestational diabetes mellitus in pregnancy is a stressful condition particularly for women and their families due to their main concern about fetus's health $(8,9)$ and mothers' awareness of the risk of diabetes makes them change their postpartum lifestyle (10).

Factors such as well-being and happiness, stress, cultural expectations and attitudes are influential in women's lifestyle changes (11). A large body of evidence suggests the impact of lifestyle changes such as proper diet and physical activity on the prevention of diabetes. Clinical guidelines highly recommend screening and treatment of gestational diabetes during pregnancy, screening of diabetes after delivery, and provision of lifestyle-related counseling (12). Accordingly, the role of training and observing the principles of proper diet during pregnancy in reducing stress and blood glucose levels was studied in the present study.

\section{Materials and Methods}

In this study, 40 pregnant women diagnosed with gestational diabetes were placed in the experimental $(n=20)$ and control $(n=0)$ groups. For data collection, a list of

Received 7 November 2015, Accepted 14 April 2016, Available online 8 May 2016

${ }^{1}$ Department of Psychology, Sari Branch, Islamic Azad University, Sari, Iran. ${ }^{2}$ Department of Endocrinology, Sari Branch, Islamic Azad University, Sari, Iran.

*Corresponding author: Ramazan Hassanzadeh, Department of Psychology, Sari Branch, Islamic Azad University, Sari, Iran. Tel: +98

9113232106, Email: R.Hasanzadeh@yahoo.com 
health centers of Sari was first compiled, and a number of centers were chosen using cluster sampling to select the subjects who were to complete the questionnaires. The necessary co-ordination was made with the authorities of the selected centers. Subjects were selected through systematic random sampling from the chosen healthcare centers.

In this study, a quasi-experimental design with pre-test and post-test control group was implemented in the format of a program developed to reduce stress among pregnant women. An experimental group and a control group participated in the research. The researcher determined the degree of effectiveness of the developed program for reducing stress levels in the experimental group and the control group did not receive any training.

First, both groups underwent a joint pre-test (using Cohen's perceived stress scale). Once the independent variable was applied (a session of proper nutrition training for diabetic pregnant women was provided for the experimental group), both groups (the experimental and the control groups) underwent the next assessment (post-test).

In data analysis, the statistical methods of mean and standard deviation/minimum and maximum scores were used for the descriptive statistics to illustrate the demographic characteristics of the groups. For the inferential statistics, analysis of covariance (ANCOVA) was used to compare the groups before and after applying the independent variable. Statistical analysis was conducted using SPSS.

\section{Results}

The mean age of participants in the experimental and control groups was 31.7 and 29.8 years, respectively. Data showed that $25 \%$ of subjects in the experimental group were younger than 30,60\% were between 30 and 35, and $15 \%$ over 35 years of age. In the control group, $50 \%$ were under $30,35 \%$ were between 30 and 35 , and $15 \%$ over 35 years of age. In other words, most subjects in both groups were under 35 years of age (Figure 1).

In the experimental group, $20 \%$ had education levels lower than a high school diploma, $20 \%$ had high school diplomas, 50\% bachelor's degrees and 10\% master's degrees. In the control group, $10 \%$ had education levels lower than a high school diploma, 20\% had high school diplomas, 15\% associate degrees, 50\% bachelor's degrees and 5\% master's degrees. In fact, most subjects had an education level higher than an associate degree (Figure 2).

Medical nutrition therapy for gestational diabetes focuses on food choices for appropriate weight gain, normoglycemia and absence of ketones. Data in Table 1 shows that nutrition training (13) is effective in reducing stress in patients with gestational diabetes and the eta value shows that the degree of effectiveness is $50 \%$. The statistical power of the test was $61 \%$, indicating the adequacy of the studied statistical samples. Therefore, the research hypothesis was confirmed at the confidence level of 95\%. Comparison of the control and experimental groups showed that the mean score in the experimental group decreased in the post-test, which suggested the effectiveness of nutri-

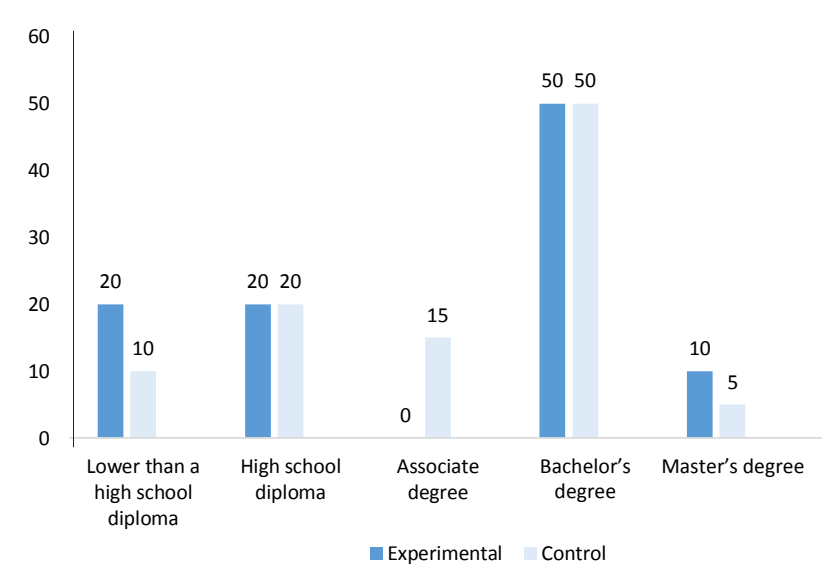

Figure 1. Frequency Distribution for Group and Educational Degree.

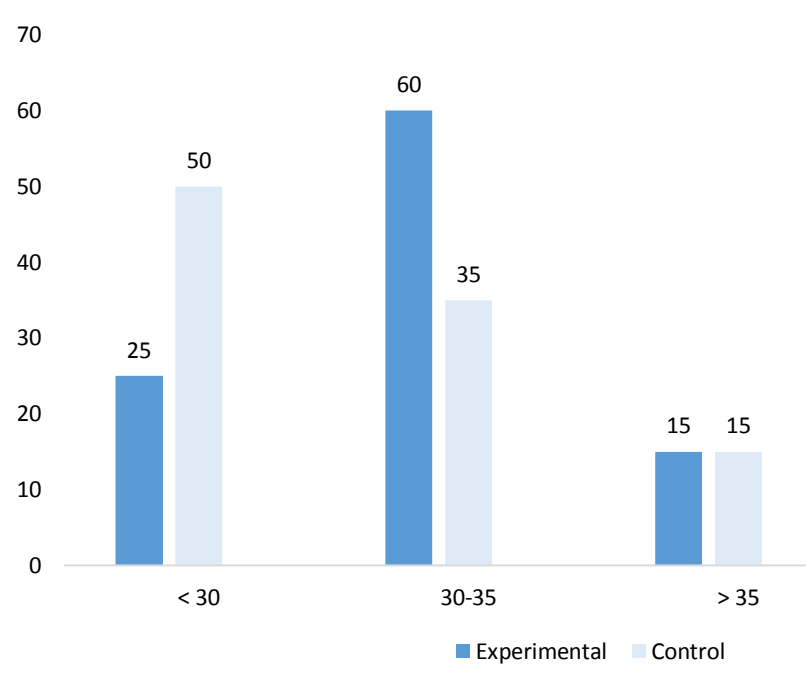

Figure 2. Frequency Distribution for Group and Age.

tion training in reducing stress in patients with gestational diabetes.

Moreover, data in Table 2 suggested that nutrition training was effective in reducing $\mathrm{HbAlC}$ in patients with gestational diabetes, with the eta value showing the degree of effectiveness as $27 \%$. The statistical power of the test was $77 \%$, indicating the adequacy of the studied statistical samples. Therefore, the research hypothesis was confirmed at the confidence level of 95\%. Comparison of the control and experimental groups showed that the mean score in the experimental group decreased in the posttest, which suggested that nutrition training was effective in reducing $\mathrm{HbA1C}$ in patients with gestational diabetes.

\section{Discussion}

In recent years, many studies from around the world have shown that changing lifestyle, promoting self-care and weight loss through continuous training reduce the stresses that are influential in the development of diabetes in women and lead to desired therapeutic effects in patients without imposing exorbitant costs on them (14).

Factors that have been mentioned in the definition of lifestyle changes include changes in diet, increased physical activity, behavioral changes associated with self-care and 
Table 1. ANCOVA Results Regarding Hypothesis 1

\begin{tabular}{lllllll}
\hline & SS & df & MS & F & $P$ & Eta2 \\
\hline Covariate & 776.08 & 1 & 776.08 & 40.67 & 0.000 & 0.52 \\
Group & 724.51 & 1 & 724.51 & 37.97 & 0.000 & 0.51 \\
Error & 705.91 & 37 & 19.07 & - & & \\
\hline
\end{tabular}

Abbreviation: ANCOVA, analysis of covariance; SS, sum of squares; MS, mean squares.

Table 2. ANCOVA Results Regarding Hypothesis 2

\begin{tabular}{llllllll}
\hline & SS & df & MS & F & $P$ & Eta2 \\
\hline Covariate & 15.92 & 1 & 15.92 & 132.19 & 0.000 & 0.78 \\
Group & 1.66 & 1 & 1.66 & 13.78 & 0.001 & 0.27 \\
Error & 4.45 & 37 & 0.12 & - & & & \\
\hline
\end{tabular}

Abbreviation: ANCOVA, analysis of covariance; SS, sum of squares; MS, mean squares.

self-control and weight loss (15).

Daniells et al assessed anxiety levels in healthy pregnant women and those with gestational diabetes. Stress levels were assessed in three phases: beginning of the third trimester, before delivery and 6 months postpartum. Results showed that stress levels at the beginning of the third trimester of pregnancy was higher in the gestational diabetes group than the healthy group; however, the differences between the groups were not significant at later stages. They stated that stress was not persistent in gestational diabetes and decreased postpartum (16). In Sri Lanka, between 2001 and 2002, a group of researchers investigated the effects of physiological stress on development of complications during pregnancy. They studied 714 pregnant women in their 12 to 28 weeks of pregnancy and measured their stress levels through using a questionnaire. The association between stress and pregnancy complications such as high blood pressure and gestational diabetes and prenatal bleeding was then studied. Results showed that factors such as gestational stress in the second trimester, body mass index, mother's weight and educational inadequacy affected the incidence of pregnancy complications (17).

In the present study, after sampling and completion of the questionnaires (using Cohen's perceived stress scale), one group received training on lifestyle changes based on nutritional status changes in a one-month course. Pre-tests before the training and post-tests were then performed and questionnaires were completed in both groups. Results indicated the positive impact of proper nutrition training on reducing stress in pregnant women, which was shown through a reduction in perceived stress scores in the experimental group compared to the control group. The effect of self-esteem on perceived stress during pregnancy and methods to deal with this stress were investigated in a study conducted by Dolatian et al (18), in which 450 pregnant women were studied in their 23-24 weeks of pregnancy. Pregnant women responded to the questions regarding perceived stress, Rosenberg's self-esteem and stress coping styles scale. Findings showed that $66.4 \%$ of participants had moderate self-esteem and 33.6\% high self-esteem. Results showed a correlation between the pregnant women's self-esteem and their perceived stress and methods of coping with stress. It was also shown that self-esteem promotion training could reduce perceived stress in pregnant women.

Similar studies evaluating stress levels in diabetics found similar results. In a study 100 pregnant women with gestational diabetes and 100 healthy pregnant women were studied to examine the impact of lifestyle changes on the incidence of gestational diabetes based on Cohen's perceived stress scale (19). It was shown that proper nutrition, self-care and stress management were effective in reducing the incidence of gestational diabetes. They stated that for every unit improvement in nutritional status, self-care and perceived stress, the incidence of gestational diabetes decreased by $6 \%, 7 \%$ and $4 \%$, respectively. Similarly, results related to self-care and perceived stress significantly differed in patients with gestational diabetes and in healthy individuals: greater self-care and perceived stress management led to further reductions in fasting blood sugar (FBS) in the experimental group. In some similar articles, however, no relationship was observed between the above-mentioned factors (20).

Javid et al investigated the effects of self-care and stress management in women with gestational diabetes and healthy pregnant women and reported similar results. They stated that healthy women had lower stress levels than those with gestational diabetes and identified stress a factor predisposing women to gestational diabetes (21). In another similar study conducted by Hamid et al (22), forty women with gestational diabetes were divided into two groups, one of which received behavioral pattern recognition training. After holding numerous classes for the experimental group, it was found that the training had a positive therapeutic effect on gestational diabetes and the experimental group had a significantly better condition than the control group regarding blood sugar control (22). Of course, some articles have stated the effect of mass media on the promotion of patients' awareness regarding selfcare and stress management. In one of the related studies, most participants changed their life style based on information available on the Internet. The authors suggested the low cost of acquiring information via the Internet and 
the difficulties of attending classes and receiving face-toface training, as the causes of inclination towards the use of the Internet as an information source (23). All these studies are somehow consistent with the present study and reflect the strong role of training in creating motivation for self-care and for making lifestyle changes.

The HbA1C test shows the blood sugar levels in a longer period (previous 6-12 weeks) (24), therefore, reduced blood sugar levels in the experimental group compared to the control group suggest that the experimental group were able to control their nutritional status and observe self-care in the long term due to explanations and practical training they received, which reflected themselves in their reduced $\mathrm{HbA1C}$ levels and consequently, in their reduced blood sugar in a longer period.

Several studies have indicated a close relationship between blood glucose and $\mathrm{HbA1C}$ levels, suggesting it as a reliable indicator of controlling gestational diabetes (24). In a research in which a questionnaire was used to study stress levels in 307 type 2 diabetes patients referred to the clinic of endocrinology and metabolism, it was shown that stress levels were higher in women who had longer history of diabetes and insulin use. These patients also had considerably higher levels of $\mathrm{HbA1C}$ (25).

\section{Conclusion}

Therefore, based on results of this study, the authors suggest the following be considered for optimal control of glucose levels in patients with gestational diabetes through stress management and lifestyle changes:

1. Given the influence of information provided in the media on the level of awareness in patients, presentation of targeted information in media in the format of long-term programs can convince patients of the necessity for selfcare and stress management.

2. Planning and creating an atmosphere of face-to-face teaching in doctors' offices and in clinics to promote self-confidence and reduce stress levels in patients.

3. Participation of nutritionists in diet changing plans.

4. Participation of psychologists in stress management.

5. Counseling patients on lifestyle changes based on the needs of diabetes patients.

\section{Ethical Issues}

All documents that were used in this article had patients consent.

\section{Conflict of Interests}

The authors declare no conflict of interests.

\section{Finnacial Support}

The researchers received no financial support or grant from any funding agency in the public and commercial sectors.

\section{Acknowledgments}

This article was extracted from a MSc. thesis in educational psychology. The authors would like to thank all profes- sors and staff of Islamic Azad University of Sari for their support.

\section{References}

1. Kim C, Tabaei BP, Burke R, et al. Missed opportunities for type 2 diabetes mellitus screening among women with a history of gestational diabetes. Am J Public Health. 2006;96(9):1643-1648.

2. Bellamy L, Casas JP, Hingorani AD, Williams D. Type 2 diabetes mellitus after gestational diabetes: a systematic review and meta-analysis. Lancet. 2009;373(9677):17731779. doi:10.1016/S0140-6736(09)60731-5.

3. Correia LL, Linhares MBM. Maternal anxiety in the preand postnatal period: a literature review. Rev Lat Am Enfermagem. 2007;15(4):677-683.

4. Baker S, Chebli M, Rees S, LeMarec N, Godbout R, Bielajew C. Effects of gestational stress: Evaluation of maternal and juvenile offspring behavior. Brain Res. 2008;1213:98-110.

5. Latendresse G. The interaction between chronic stress and pregnancy: preterm birth from a biobehavioral perspective. J Midwifery Womens Health. 2009;54(1):8-17. doi:10.1016/j.jmwh.2008.08.001

6. Cohen S, Kessler RC, Gordon LU. Strategies for measuring stress in studies of psychiatric and physical disorders. Measuring stress: A guide for health and social scientists. 1995 Jan 5.

7. WHO. Facing the Challenges, Building Solution. Report from the WHO European Ministerial Conference. The Regional Office for Europe of the World Health Organization. http://www.euro.who.int/_data/assets/pdf_ file/0008/96452/E87301.pdf. Accessed January 21, 2011.

8. Persson M, Winkvist A, Mogren I. 'From stun to gradual balance'-women's experiences of living with gestational diabetes mellitus. Scand J Caring Sci. 2010;24(3):454-462. doi:10.1111/j.1471-6712.2009.00735.x.

9. Evans MK, O'Brien B. Gestational diabetes: the meaning of an at-risk pregnancy. Qual Health Res. 2005;15(1):66-81. doi:10.1177/1049732304270825.

10. Evans MK, Patrick LJ, Wellington CM. Health behaviours of postpartum women with a history of gestational diabetes. Can J Diabetes 2010;34(3):227-232. doi:10.1016/S14992671(10)43011-7.

11. Bandyopadhyay M, Small R, Davey MA, Oats JJ, Forster DA, Aylward A. Lived experience of gestational diabetes mellitus among immigrant South Asian women in Australia. Aust N Z J Obstet Gynecol. 2011;51(4):360-364. doi:10.1111/j.1479-828X.2011.01322.x.

12. Canadian Diabetes Association. Canadian Diabetes Association 2013 Clinical Practice Guidelines for the Prevention and Management of Diabetes in Canada. Can J Diabetes. 2013;37(suppl 1):S1-S212.

13. Franz MJ, Bantle JP, Beebe CA, et al. Nutrition principles and recommendations in diabetes. Diabetes Care. 2004;27(suppl 1):S36-46.

14. Heydari GH, Moslemi S, Montazerifar F, Heydari M. Efficacy of education on knowledge, attitude \& practice of Type II diabetic patients in relation to correct nutrition. Zahedan J Res Med Sci (TABIB-E-SHARGH). 2003;4(4):207-213.

15. Gabbe SG, Landon M, Warren-Boulton E, Fradkin J. Promoting health after gestational diabetes: A national diabetes education program call to action. Obstet Gynecol. 2012;119(1):171-176. doi:10.1097/ AOG.0b013e3182393208. 
16. Daniells S, Grenyer BF, Davis WS, Coleman KJ, Burgess JA, Moses RG. Gestational Diabetes Mellitus Is a diagnosis associated with an increase in maternal anxiety and stress in the short and intermediate term? Diabetes Care. 2003;26(2):385-389. doi:10.2337/diacare.26.2.385.

17. Abeysena C, Jayawardana P, Seneviratne R. Effect of psychosocial stress on maternal complications during pregnancy: a cohort study. Int J Collaborative Res Internal Med Public Health 2010;2:436-48.

18. Dolatian M, Mirabzadeh A, Forouzan AS, et alZ. Correlation between self-esteem and perceived stress in pregnancy and ways to coping with stress. Pajoohandeh J. 2013;18(3):148155.

19. Javid FM, Simbar M, Dolatian M, Majd HA. Comparison of lifestyles of women with gestational diabetes and healthy pregnant women. Global J Health Sci. 2015;7(2):162-169.

20. Silveira ML. Psychosocial stress in pregnancy and risk of gestational diabetes in hispanic women. Paper presented at: The 141st APHA Annual Meeting and Exposition; 2013 Nov 2-6; Bostan, USA.

21. Momeni JF, Simbar M, Dolatian M, Alavi Majd $\mathrm{H}$.
Comparison of pregnancy self-care, perceived social support and perceived stress of women with gestational diabetes and healthy pregnant women. Iran J Endocrinol Metab 2014;16(3):156-164.

22. Hamid N. Effects of stress management training on glycemic control in women with type 2 diabetes. Iran J Endocrinol Metab. 2011;13(4):346-353.

23. Nicklas JM, Zera CA, Seely EW, Abdul-Rahim ZS, Rudloff ND, Levkoff SE. Identifying postpartum intervention approaches to prevent type 2 diabetes in women with a history of gestational diabetes. BMC Pregnancy Childbirth 2011;11:23. doi:10.1186/1471-2393-11-23.

24. Gandhi RA, Brown J, Simm A, Page RC, Idris I. HbA1c during pregnancy: its relationship to meal related glycaemia and neonatal birth weight in patients with diabetes. Eur J Obstet Gynecol Reprod Biol. 2008;138(1):45-48. doi:10.1016/j.ejogrb.2007.08.011.

25. Eom YS, Park HS, Kim SH, et al. Evaluation of stress in Korean patients with diabetes mellitus using the problem areas in Diabetes-Korea questionnaire. Diabetes Metab J. 2011;35(2):182-187. doi:10.4093/dmj.2011.35.2.182.

Copyright (c) 2016 The Author(s); This is an open-access article distributed under the terms of the Creative Commons Attribution License (http://creativecommons.org/licenses/by/4.0), which permits unrestricted use, distribution, and reproduction in any medium, provided the original work is properly cited. 